\title{
Secure Communication Over Radio Channels
}

\author{
Shlomi Dolev \\ Ben-Gurion University \\ Beer-Sheva, Israel \\ dolev@cs.bgu.ac.il \\ Rachid Guerraoui \\ EPFL IC \\ Lausanne, Switzerland \\ rachid.guerraoui@epfl.ch
}

\author{
Seth Gilbert \\ EPFL IC \\ Lausanne, Switzerland \\ seth.gilbert@epfl.ch \\ Calvin Newport
MIT CSAIL
Cambridge, MA, USA
cnewport@mit.edu
}

\section{INTRODUCTION}

In recent years, wireless networking technology has held out the prospect of enabling communication without the need for physical network infrastructure. Protocols such as Bluetooth realize this promise by allowing ad hoc collections of nearby devices to easily construct a simple one-hop network (sometimes called a piconet). The radio medium, however, is publicly accessible. A committed malcontent can eavesdrop, interfere with broadcasts, and spoof messages. Shared secrets provide a standard mechanism for circumventing these types of attacks. If the honest devices share a secret key that is unknown to the adversary, they can eliminate eavesdropping and message spoofing by encrypting and signing their messages, respectively. Another common use of a shared secret key is to generate a pseudo-random channelhopping pattern that allows the participants to efficiently avoid adversarial interference.

This approach, however, begs an obvious question: How do we establish shared secrets? Protocols such as Bluetooth, for example, require that a shared secret is manually entered into the relevant devices (in the form of a passkey). This might prove inconvenient or impossible in many situations. Furthermore, even when pre-programming is feasible, it might be useful to be able to re-key dynamically, for example, after the detection of a compromised device. Another approach is to rely on trusted third-party infrastructure, such as a PKI, to authenticate and secure communication. In many settings, however, such services are unavailable.

\section{Authenticated Message Exchange}

In this paper we address this crucial question. We begin by studying the problem of Authenticated Message Exchange (AME). Initially, we are given an arbitrary set $E$ of pairs of devices that want to exchange information. The goal of AME is for as many of these pairs as is possible to communicate messages in an authenticated manner. For the purpose of this paper, we assume that there are $t+1$ channels available for communication and that the adversary can affect up to $t$ channels per round, causing interference or spoofing messages. (Notice that this is the minimal number of channels for which any communication is feasible.)

A significant challenge lies in efficiently achieving authenticated communication. A deterministic algorithm can read-

\begin{abstract}
Permission to make digital or hard copies of all or part of this work for personal or classroom use is granted without fee provided that copies are not made or distributed for profit or commercial advantage and that copies bear this notice and the full citation on the first page. To copy otherwise, to republish, to post on servers or to redistribute to lists, requires prior specific permission and/or a fee.

PODC'08, August 18-21, 2008, Toronto, Ontario, Canada.

Copyright 2008 ACM 978-1-59593-989-0/08/08 ...\$5.00.

Wireless Radio Networks, Malicious (Byzantine) Interference, Randomized Algorithms
\end{abstract}


ily achieve authentication by relying on a pre-determined broadcast schedule: If $p_{i}$ is known to be broadcasting on a given channel in a given round, the worst the adversary can do is broadcast concurrently and cause a collision; if another node $p_{j}$ receives a message on that channel in that round, it can conclude that the message was sent by $p_{i}$ itself, not the adversary. A purely randomized approach, on the other hand, is difficult to authenticate since the receiver cannot determine whether the adversasry or the honest node was broadcasting on that channel: with some probability the honest node sent the message, but with some probability the honest node was on a diffrent channel, allowing the adversary to spoof messages. Unfortunately, we conjecture that a purely deterministic solution would require exponential time to solve AME. In this paper we identify a middle-ground: harnessing the efficiency of randomization for transmitting data, while leveraging deterministic broadcast scheduling to authenticate the messages.

We describe a randomized protocol called f-AME (fastAuthenticated Message Exchange) that solves the AME problem with optimal resilience: all but at most $t$ devices successfully communicate as specified by the set $E$. The f-AME protocol requires no pre-shared secrets or trusted third-party infrastructure. It terminates in $O\left(|E| t^{2} \log n\right)$ rounds. By contrast, relying on an all-to-all gossip protocol, as can be found in [13], would result an exponential running time, and yet still fail to achieve optimal resilience.

The core insights behind $\mathrm{f}-\mathrm{AME}$ are graph theoretic. To best capture this connection, we first define an abstract graph-theoretic game that we call Starred-Edge Removal. We then describe a greedy strategy that efficiently solves the game. Our final f-AME protocol simulates the game on the multi-channel network, using a randomized feedback routine to synchronize the distributed view of the game and maintain a correspondence between the game state and the AME message set. We show that our efficient strategy for the edge-removal game induces an optimally resilient solution to the AME problem.

\section{Established a Secret Group Key}

We then use f-AME to establish a shared secret group key. The algorithm begins with a setup phase in which secure pairwise keys are established. We initialize f-AME with messages generated by a one-round cryptographic key-exchange protocol (such as Diffie-Hellman) and a set $E$ of pairs describing a sparse $t+1$-connected graph with $n(t+1)$ edges that we call a " $(t+1)$-leader spanner." The resulting pairwise shared keys are used to distribute up to $t+1$ proposals for the shared group key. An agreement protocol allows the nodes to safely agree on a single proposal to adopt. The total running time to establish the shared group key is $O\left(n t^{3} \log n\right)$ rounds.

\section{Long-Lived Communication Service}

Shared secret group keys have a variety of uses. In this paper we describe one: the construction of a secure, reliable, and authenticated long-lived communication service. Once a group key is established, the service requires only $\Theta(t \log n)$ rounds for an arbitrary pair to communicate. It differs from $\mathrm{f}-\mathrm{AME}$ in that it is long-lived, rather than single-shot. Moreover, after the setup phase (which relies on f-AME to establish a group key), the nodes can exchange messages more efficiently than re-running the f-AME protocol. Also unlike
f-AME, any pair can communicate whenever it chooseseven if no other nodes are interested in communicating at that time. (By contrast, $\mathrm{f}-\mathrm{AME}$ relies on the fact that many pairs want to communicate, thus preventing the adversary from blocking all of them.) This represents, to the best of our knowledge, the first such communication primitive for a radio network with malicious interference and no a priori shared secrets or trusted infrastructure.

\section{RELATED WORK}

There exists much research in broadcast and gossip in the context of single-channel radio networks (e.g., [1-3, 7, 8, 11, $15,18,20,23])$. Much of this research focuses on the problem of channel contention, and assumes reliable devices in a non-malicious environment. Recently, there has been some interest in crash-tolerance in radio networks (e.g., $[9,10,19]$ ), and Byzantine-resilient broadcast in radio networks $[4,16]$. In these latter studies, however, the adversary cannot disrupt communication. When adversarial disruption is allowed, there exist three common approaches in the literature. The first assumes that messages may be corrupted at random (e.g., [21]). The second bounds the number of messages that the adversary can transmit or disrupt, due, for example, to a limited energy budget (e.g., [14,17]). The third assumes some manner of shared secrets. There have been proposed a variety of approaches for establishing such a shared secret, all of which require some form of human intervention (e.g., the manually configured passkeys of Bluetooth [5]), or out-of-band communication (e.g., physical contact between devices [22], a limited-bandwidth secure sidechannel [6,12], location information [6] and strong collision detection $[6,14])$.

The present paper, along with [13], are the first, to our knowledge, to consider multi-channel networks subject to malicious disruption in which processes do not possess a priori shared secrets (or have access to out-of-band means for establishing these secrets). Dolev et al. [13] consider the problem of almost gossip, in which all but $t$ rumors are delivered to all but $t$ nodes. They focus on oblivious algorithms that do not adapt to the execution in progress, and show for the special case of $t=1$ that there exists a tight bound for gossip of $\Theta\left(n^{2} / C^{2}\right)$. They extend their algorithm for general $t$, achieving running time $O\left((e n / t)^{t+1}\right)$. Thus using gossip to solve AME would be quite slow, and also would only achieve suboptimal resilience, i.e., $2 t$-disruptability (see Section 4 for more details).

\section{MODEL}

We assume a single-hop radio network with $n$ devices (which we call "nodes"), described by $\Pi=\left\{p_{1}, \ldots, p_{n}\right\}$. The network has $C>1$ communication channels. Executions proceed in synchronous rounds; all nodes start in the same round. During each round, each node can choose to transmit or receive on a single channel. If a single node transmits on a channel, all receivers on that channel receive the transmission. If no node, or two or more nodes transmit on the same channel, then the receivers on that channel receive nothing. (We do not assume that nodes can detect collisions.)

We also assume the presence of an adversary that can transmit on up to $t<C$ channels during each round, and can listen on all $C$ channels. The adversary can therefore disrupt communication in two ways: (1) jamming: by transmitting 
concurrently with an honest node, the adversary causes a message to be lost due to collision; and (2) spoofing: by transmitting a fake message on an otherwise empty channel, the adversary causes a node to receive incorrect information. Notice that since communication is not authenticated, a node cannot definitively determine who sent a given message. As is typical, we assume the adversary does not know in advance the honest nodes' random choices; only by observing a node's actions is it possible to determine which random choice it made. Without loss of generality, we assume that at the end of each round, the adversary learns all random choices made in all completed rounds.

\section{PRELIMINARIES}

In this paper, we focus on the case where $C=t+1$; notice that this is the minimum number of channels for which any communication is possible. Where relevant, we briefly discuss how our results can be improved for larger values of $C$, specifically, the case where $C=2 t$. We also fix $n$ to be sufficiently large with respect to $t$, specifically $n>3(t+1)^{2}+2(t+1)$. (This is not unreasonable, since $n$ is typically large with respect to the number of channels $C$, and $t<C$.)

The focus of this paper is a problem we call Authenticated Message Exchange (AME). An AME protocol is initially provided with a set $E$ of ordered pairs. For each ordered pair $(v, w) \in E$ we attempt to send a message $m_{v, w}$ from $v$ to $w$. The AME protocol guarantees three properties: (1) authenticity, i.e., a node receives only authentic messages and successfully ignores messages spoofed by the adversary; (2) sender awareness, i.e., each node knows which of their messages were successfully received; and (3) limited disruptability, i.e., the adversary can disrupt only a limited number of the nodes that want to communicate. More formally, an AME protocol makes the following guarantees:

Definition 1 ( $d$-DisRuptable AME Protocol $).$ For $d$ a non-negative integer, we say that protocol $\mathcal{P}$ is a $\boldsymbol{d}$-disruptable $\boldsymbol{A} \boldsymbol{M E}$ protocol if for every set $E$ of ordered pairs of distinct nodes from $\Pi$, where $|E| \geq d$, and for every $(v, w) \in E$, message $m_{v, w}$ is initially known only to $v$, the following properties hold with high probability:

1. Authentication: For each $(v, w) \in E: w$ outputs either $\langle(v, w)$, fail $\rangle$ or $\left\langle(v, w), m_{v, w}\right\rangle$.

2. Sender Awareness: For each $(v, w) \in E: v$ can determine whether $j$ output $\langle(v, w)$, fail $\rangle$ or $\left\langle(v, w), m_{v, w}\right\rangle$.

3. $d$-Disruptability: Let $E^{\prime} \subseteq E$ be the subset of pairs that output fail. The minimum vertex cover of the disruption graph $G_{d}=\left(\Pi, E^{\prime}\right)$ contains no more than $d$ vertices.

Notice that the disruptability property describes the number of "failures" that can be induced by the adversary. That is, for a $d$-disruptable protocol, there exists a set of no more than $d$ nodes such that if we remove these nodes from consideration, all other nodes succeed in communicating their messages. We observe that no protocol can achieve better than $t$-disruptability; this implies that the adversary can always prevent at least $t$ of the nodes from communicating successfully. Notice that in the case of deterministic AME protocols, this is immediately clear since the adversary can readily target $t$ of the nodes; slightly more care is needed in the case of randomized protocols, but the same argument holds.

Theorem 2. For $n \geq 2 t$ and $d<t$ : there exists no $d$ disruptable Authenticated Message Exchange protocol.

Proof. Assume for the sake of contradiction that such a protocol $\mathcal{P}$ exists. Fix $E=\{(i, i+t) \mid i \in\{1, \ldots, t\}\}$, that is, there are $t$ disjoint pairs of nodes that want to communicate. Consider an execution $\alpha_{1}$ of $\mathcal{P}$ in which the adversary simulates nodes 1 through $t$ and attempts to disseminate a fake message (different from the real message, in each case); whenever a node $i \in\{1, \ldots, t\}$ broadcasts a message on a deterministically chosen channel, the adversary does the same; whenever $i$ broadcasts a message on a randomly chosen channel, the adversary also chooses a channel at random based on the same distribution. Because the adversary can broadcast on up to $t$ different channels per round, it can carry out this simulation faithfully.

Property (3) of the AME definition requires that at least one pair in $E$ does not output fail, since $|E|=t$ and $d<$ $t$. Without loss of generality, assume this is pair $(1, t+$ 1). Property (1) requires that, with high probability, $t+1$ outputs $m_{1, t+1}$. Let $r_{1}$ be the string of random bits used by process 1 , and let $r_{A}$ be the string of random bits used by the adversary. To process $t+1$, however, this execution $\alpha_{1}$ is indistinguishable from another execution $\alpha_{2}$ in which the adversary chooses random bits $r_{1}$ and process 1 chooses random bits $r_{A}$. Moreover, both executions $\alpha_{1}$ and $\alpha_{2}$ occur with equal probability. It follows, therefore, that node $t+$ 1 has equal probability of outputting the correct message, $m_{1, t+1}$, and the fake message generated by the adversary. This violates property (1), implying a contradiction.

\section{AUTHENTICATED MESSAGE EXCHANGE}

In this section, we develop an Authenticated Message Exchange (AME) protocol with optimal resilience, i.e., the protocol is at most $t$-disruptable. Throughout this section, fix $G^{A M E}=\left(\Pi, E^{A M E}\right)$ to be the directed graph representing the pairs that have messages to exchange, and let $n=|\Pi|$. For every $(v, w) \in E^{A M E}$, let $m_{v, w}$ be the message that $v$ is sending to $w$. We call our protocol $\mathrm{f}$-AME (fast-Authenticated Message Exchange) as it runs in time $O\left(\left|E^{A M E}\right| t^{2} \log n\right)$ rounds. Our f-AME protocol is built on two insights.

The first insight is related to authentication: a good method for achieving authenticated communication is to schedule $t+1$ sender/receiver pairs to communicate concurrently; each sender then transmits its message directly to the receiver. The adversary cannot simultaneously block all $t+1$ channels, and hence at least one message is successfully communicated. If the channel assignment is determined deterministically and in advance, then the adversary can disrupt communication but not spoof messages, as any broadcast by the adversary will simply cause a collision.

This strategy alone, in which each message is transmitted directly from the source to the destination, can achieve no better than $2 t$-disruptability. Consider a complete communication graph. The adversary can identify $t$ disjoint sets of three processes; it can block any channel which contains two or more processes from the same set of three. The result: a disruption graph with $t$ edge-disjoint triangles, inducing minimum vertex cover of size $2 t$. 
We turn, therefore, to our second insight: to further reduce disruptability further, a node can recruit surrogates to relay messages on its behalf. These surrogates later allow us to schedule two edges to broadcast simultaneously, even if they share a source. (Notice that by routing messages via surrogates, the adversarial strategy of isolating triangles, as described above, is no longer feasible.)

\section{Roadmap}

To best clarify our deployment of these insights, we present our protocol in stages. First, in Section 5.1, we define an abstract graph-theoretic "game" that we call starred-edge removal. This game isolates the core scheduling concerns of removing edges from the disruption graph and recruiting surrogates from the more practical concerns of coordinating distributed nodes on a multi-channel network. In Section 5.2, we describe an efficient strategy, greedy-removal, that solves the starred-edge removal game in a minimal number of rounds. In the final two sections, Sections 5.3 and 5.4, we describe a distributed simulation of the starred-edge removal game that maintains a correspondence between the game graph and the AME disruption graph. At the core of the distributed simulation is a randomized sub-routine, communication-feedback, that informs all nodes, with high probability, which channels were disrupted during a given round.

\subsection{The $(G, t)$-starred-edge removal game}

In this section, we define the $(G, t)$-starred-edge removal game, where $G=(V, E)$ is a directed graph and $t$ is a natural number less than $n=|V|$. As a point of notation, for every edge $(v, w) \in E$, we refer to $v$ as the source and $w$ as the destination. Throughout the game, we also maintain an auxiliary set $S \subseteq V$, initially empty. (If a node $v \in V$ is in the set $S$, we say that $v$ is starred.) The game consists of a series of rounds. In each round:

1. The player proposes a set $P$ that is comprised of the following: (1) nodes from $V$; and (2) edges from $E$. The proposal must satisfy the proposal restrictions described below.

2. The referee responds by choosing a non-empty subset of nodes and edges from $P$.

3. For every node chosen by the referee, the player adds the node to $S$. For every edge chosen by the referee, the edge is removed from $E$.

The game terminates if the player succeeds in removing enough edges from $G$ to produce a new graph with a vertex cover of size at most $t$. The goal of the referee, by contrast, is to delay the player from completing the game for as long as possible.

\section{Proposal Restrictions}

It remains to specify the restrictions on the player's proposed set $P$ of nodes and edges. They are as follows:

1. The set $P$ must consist of exactly $t+1$ items, each of which is either a node in $V$ or an edge in $E$.

2. Every node in $P$ is unique, i.e., it does not appear as either the source or destination of any edge in $P$.
3. No two edges in $P$ share a destination.

4. Two edges in $P$ share a source $v \in V$ only if $v \in S$.

For example, if $v, w, z$ are nodes in $V$, Restriction 3 prohibits the player from proposing both edges $(v, z)$ and $(w, z)$. Similarly, Restriction 4 prohibits the player from proposing both edges $(v, w)$ and $(v, z)$ unless node $v \in S$. Roughly speaking, a node being added to $S$ corresponds to the fact that it has successfully recruited surrogates to broadcast on its behalf (which is required to achieve $t$-resilience, as per our discussion above).

\subsection{The Greedy Removal Strategy}

In this section, we describe a centralized strategy, which we call greedy-removal, that solves the $(G, t)$-starred-edge removal game in $O(|E|)$ rounds. Our strategy ensures that in each round of the game, either a new edge is removed from $E$ or a new node is added to $S$. The greedy-removal strategy works as follows. Define two sets $P_{1}$ and $P_{2}$, with respect to graph $G=(V, E)$ :

- $P_{1}=\{v \in V \backslash S:(v, *) \in E\}$. That is, $P_{1}$ consists of the set of nodes not in $S$ that are the source of some edge in $E$.

- $P_{2}=\left\{(v, w) \in E: v, w \notin P_{1}\right\}$. That is, $P_{2}$ consists of edges in which neither source nor destination is in $P_{1}$. This implies that if $(v, w) \in P_{2}$, then $v \in S$.

The player chooses any arbitrary subset of $t+1$ elements from $P_{1} \cup P_{2}$ that satisfy Proposal Restrictions 1-4 of the $(G, t)$-starred-edge removal game. If there is no subset of $P_{1} \cup P_{2}$ of size at least $t+1$ that satisfies Restrictions 14 , we say that the greedy-removal strategy has terminated. The key result in this section is that the greedy-removal strategy terminates only if the minimum vertex cover of $G$ is no greater than $t$; this implies that the game is complete.

Lemma 3. Assume that no subset $P \subseteq P_{1} \cup P_{2}$ satisfies Restrictions 1-4 of the $(G, t)$-starred-edge removal game. Then graph $G$ has a minimum vertex cover no larger than $t$.

Proof. We proceed in the following three steps: (1) constructing a set $V^{\prime} \subseteq V ;(2)$ arguing that it is of size at most $t$; and (3) showing that it is a vertex cover for $G$. We define the set of vertices $V^{\prime}$ as follows:

$$
V^{\prime}=P_{1} \cup\left\{w:(*, w) \in P_{2}\right\}
$$

Every node in $P_{1}$ is included in the set $V^{\prime}$; in addition every destination for every edge in $P_{2}$ is also included in $V^{\prime}$. We first argue that the number of destinations in $P_{2}$ is at most $t-\left|P_{1}\right|$; we can derive from this that $\left|V^{\prime}\right| \leq t$. Assume for the sake of contradiction that there exist at least $t-\left|P_{1}\right|+1$ destinations in $P_{2}$. We can define the following proposal $P$ of size $t+1$ : all the nodes in $P_{1}$, along with the $t-\left|P_{1}\right|+1$ destination-disjoint edges from $P_{2}$. It follows immediately that this set satisfies Restriction 1. Restriction 2 follows from the fact that every edge in $P_{2}$ is disjoint from every node in $P_{1}$. Restriction 3 follows from the fact that we have chosen at most one edge in $P_{2}$ for each destination. Restriction 4 is satisfied by the fact the source of every edge in $P_{2}$ is also in $S$. (Otherwise, the source of the edge would be included in $P_{1}$ ). The existence of this proposal $P$ of size $t+1$ contradicts our assumption that the player has no legal 


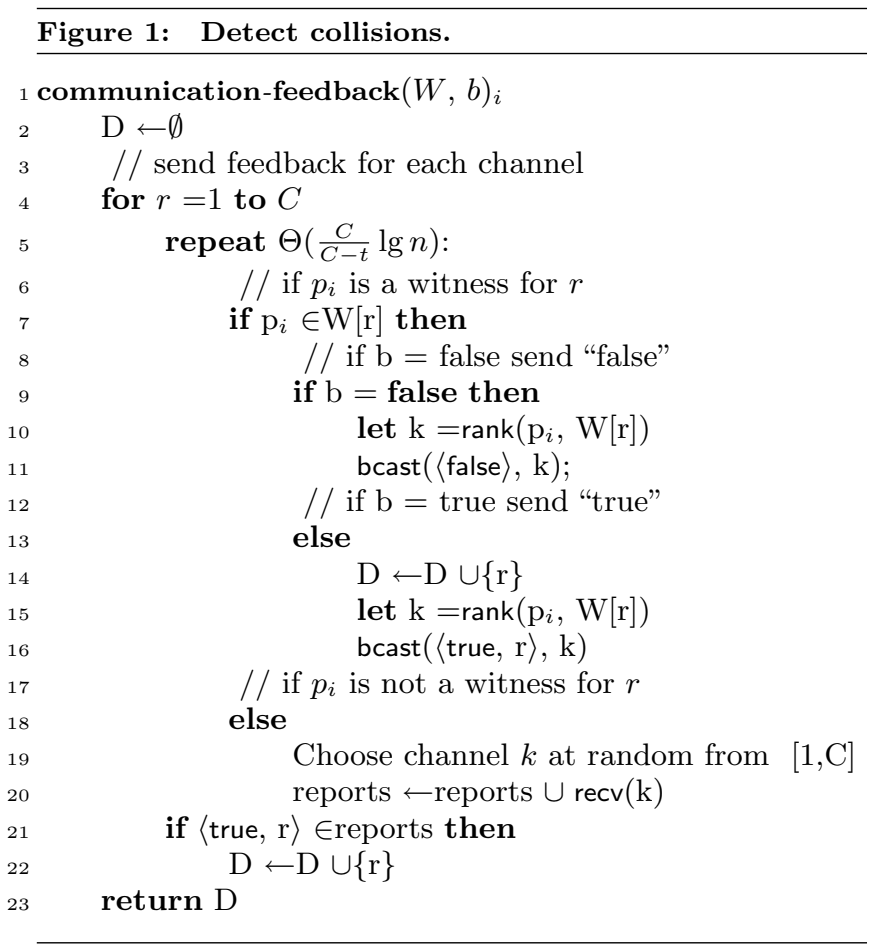

move available, from which we conclude that $V^{\prime}$ is of size at most $t$.

Finally, we argue that $V^{\prime}$ is a vertex cover for $G$. In particular, consider some edge $(v, w) \in E$. If $(v, w) \in P_{2}$, then, by definition, $w \in V^{\prime}$. Therefore, $(v, w)$ is covered by $V^{\prime}$. Assume, then, that $(v, w) \notin P_{2}$. By the definition of $P_{2}$ : either $v$ or $w$ must therefore be in $P_{1}$. This implies however that either $v$ or $w$ is in $V^{\prime}$, once again covering the edge. Thus, we have demonstrated a vertex cover of size at most $t$.

THEOREM 4. The greedy-removal strategy solves the $(G, t)$ starred-edge removal game in $O(|E|)$ moves.

Proof. If the referee returns an edge from $P_{2}$, this removes an edge from $E$. If the referee returns a node from $P_{1}$, this adds a new node to $S$ (as every node in $P_{1}$ is not in $S$ ). Since at most $|E|$ edges can be removed from $E$, and at most $2|E|$ nodes can be added to $S$, the desired bound holds.

\subsection{Communication Feedback}

Our f-AME protocol simulates the starred-edge removal game. A key sub-routine needed by this simulation is a method for nodes to agree on which channels were disrupted during a given round. The communication-feedback protocol satisfies this need.

Specifically, after executing a round of communication, the nodes call the feedback routine communication-feedback in order to agree on which channels have been disrupted; the feedback routine takes two parameters: (1) $W$, a partition of the processes $\left\{p_{1}, \ldots, p_{C^{2}}\right\}$ into $C$ sets of size $C ;(2) b$, a binary flag, either true or false. Intuitively, $W$ assigns as set of $C$ "witnesses" to each of the $C$ channels, and the flag $b$ indicates whether the caller of the feedback routine has received a message in that round of communication.

We assume that all processes call communication-feedback in the same round with the same partition $W$. For each $c \in\{1, \ldots, C\}$, we refer to the processes $W[c]$ as witnesses for channel $c$. We also assume that every witness for channel $c$ has the same value of the flag $b$, which we refer to as the channel $c$ flag. For every $c \in\{1, \ldots, C\}$, let $b_{c}$ describe the channel $c$ flag. We show that under these circumstances, the call to communication-feedback satisfies the following:

LEMMA 5. Each invocation of communication-feedback terminates in time $O\left(t^{2} \log n\right)$, and returns a set $D$ satisfying the following property, with high probability: for every channel $c \in\{1, \ldots, C\}, c \in D$ if and only if $b_{c}=$ true.

Proof. Fix come channel $c \in\{1, \ldots, C\}$, and some node $p_{j} \in \Pi$ that invokes communication-feedback. It is clear by inspection that the feedback routine terminates in $C \cdot \frac{C \log n}{C-t}$ rounds, which is $O\left(t^{2} \log n\right)$. We now examine the set $D$ produced when $p_{j}$ calls communication-feedback.

First, assume that $b_{c}=$ false. Consider the iteration of lines 4-22 where $r=c$, as this is the only instance in which $c$ can be added to the set $D$. Notice that in each of the $\Theta(t \log n)$ rounds that take place where $r=c$, for each channel $k \in\{1, \ldots, C\}$, one of the witnesses in $W[c]$ broadcasts a false message on channel $k$ (line 11). Thus it is impossible for $p_{j}$ to receive a $\langle$ true, $c\rangle$ message, and hence $c \notin D$ for node $p_{j}$. (Notice, the adversary can broadcast $\langle$ true, $c\rangle$, but because every channel is occupied by a broadcasting witness, this will lead only to a collision.)

Consider instead the case in which the value $b_{c}=$ true. If $p_{j}$ is a witness for $c$, then $p_{j}$, immediately adds $r$ to $D$ (line 14). Otherwise, we will argue that, with high probability, $p_{j}$ receives a $\langle$ true, $c\rangle$ message from some witness for channel $c$. Specifically, for each of the $\Theta\left(\frac{C}{C-t} \log n\right)$ rounds that take place where $r=c$, one witness on each channel is broadcasting $\langle$ true, $c\rangle$. The process $p_{j}$ chooses a random channel on which to listen (line 20). As a result, we note that in each round, $p_{j}$ has probability no worse than $(C-t) / C$ of selecting a channel not disrupted by the adversary and therefore receiving a $\langle$ true, $c\rangle$ message. Receiving this message causes $p_{j}$ to add $c$ to the set $D$.

It follows that over $\Theta\left(\frac{C}{C-t} \log n\right)$ rounds, we can conclude, by a straightforward Chernoff bound that $p_{j}$ receives $\langle$ true, $c\rangle$, and thus adds $c$ to $D$, with high probability. By a union bound over all nodes, we conclude that every $p_{j} \in \Pi$ that is not a witness receives such a message with high probability.

\subsection{The fast-AME Protocol}

Here were bring together all the pieces to present $\mathrm{f}-\mathrm{AME}$ (fast-Authenticated Message Exchange). The protocol is $t$ disruptable and completes in $O\left(|E| t^{2} \log n\right)$ rounds. At the core of our protocol is a distributed simulation of the $(G, t)$ starred-edge removal game, where, initially, $G=G^{A M E}$. The simulation maintains a graph equivalence invariant: after $r$ simulated rounds of the game, the graph $G$ in the game is equivalent to the current disruption graph. Since the starred-edge removal game terminates only when the vertex cover is no greater than $t$, the game simulation results in $t$-disruptability.

\section{Overview:}

The f-AME protocol, described at a high level in Figure 2, proceeds by simulating moves in the starred-edge removal game. Each move is simulated using $\Theta\left(t^{2} \log n\right)$ rounds of communication, and consists of two phases: a message- 


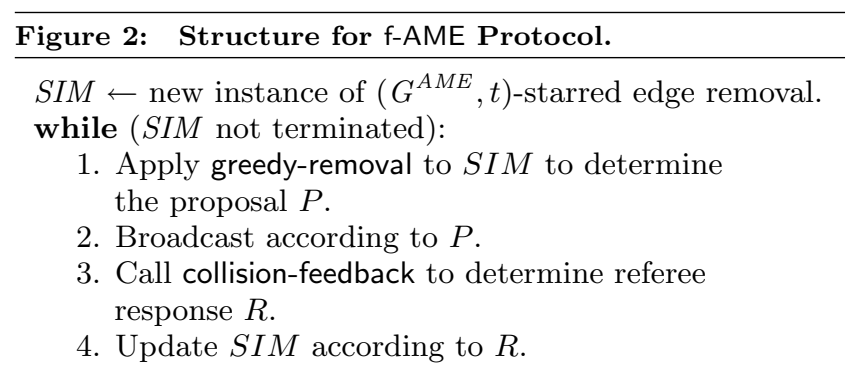

transmission phase (which requires one round of communication), and a feedback phase (which requires $\Theta\left(t^{2} \log n\right)$ rounds of communication). In the first phase, the nodes simulate the player's proposal in the game, choosing a set $P$ and transmitting messages accordingly. In the second phase, the nodes simulate the referee, using the communication-feedback routine to agree on the set returned by the referee.

\section{State:}

Each node $p_{j} \in \Pi$ maintains the following state throughout the execution of the protocol: a graph $G_{j}=\left(V_{j}, E_{j}\right)$; and a set $S_{j}$. These represent $p_{j}$ 's local copy of the starrededge removal game. Initially, for every $p_{j}, G_{j}=G^{A M E}$ and $S_{j}=\emptyset$.

\section{Message-transmission phase:}

The message transmission phase proceeds as follows. Each node $p_{j}$ locally applies the greedy-removal strategy to its local copy of $G_{j}$ and $S_{j}$ to determine a valid proposal $P_{j}$. We will argue in our correctness proof that every node generates the same proposal $P_{j}$, and hence all the nodes behave consistently in the rounds that follows. We now describe how a node calculates which nodes should broadcast or listen during the message-transmission phase, and which channels these nodes should use. Notice that given $P_{j}$, node $p_{j}$ can determine the precise behavior of every other node, and thus can determine the appropriate action to take during the message-transmission phase.

Recall that $P_{j}$ consists of both nodes and edges. Each node in $P_{j}$ is scheduled for one of the $t+1$ channels on which to broadcast during the message-transmission phase. Ideally, we would also schedule each edge in $P_{j}$ on one of the $t+1$ channels: for each edge, the source would choose a channel on which to broadcast, and the destination would listen on the same channel.

It may, however, be the case that some of the edges in $P_{j}$ share the same source, and hence cannot be scheduled simultaneously. We know, however, by the restrictions of the starred-edge removal game that none of the edges in $P_{j}$ share the same destination; nor do any of the edges in $P_{j}$ contain a node in $P_{j}$.

Consider the case where $P_{j}$ includes two edges that share a source, say $(v, w)$ and $(v, z)$. In this case, we know that $v \in S$, by the restrictions of the game. We will show that every node in $S$ has at least $3(t+1)$ surrogates. Since there are at most $2(t+1)$ nodes involved with $P_{j}$ as either a node, a source, or a destination, we can conclude that there are at least $t+1$ nodes available to act as surrogates for $v$. Thus, for every edge in $P_{j}$, either the source or one of its available surrogates is scheduled to broadcast on a channel, and we schedule the destination to receive on the same channel.

Lastly, $3(t+1)$ witnesses are scheduled to listen on each of the $t+1$ channels that are being used. Since $n>3(t+1)^{2}+$ $2(t+1)$, and since at most $2(t+1)$ nodes have, to this point, been assigned either to broadcast or to listen, it is clear that there are enough nodes to satisfy this assignment. Of these witnesses, for each channel $c \in\{1, \ldots, C\}$, we choose a subset of $t+1$ nodes per channel and assign these to $W[c]$ for use in the call to communication-feedback that occurs during the next phase.

To this point, we have described the choice of nodes to broadcast and listen in a centralized fashion as a function of $P_{j}$. Node $p_{j}$ behaves according to the schedule which it calculates locally from $P_{j}$. As we show that every node calculates the same $P_{j}$, we can conclude that every node calculates the same scheduling of channels, thus leading to consistent behavior that avoids contention.

Thus, to summarize a node's behavior in the messagetransmission phase: each node $p_{j}$ examines the schedule that has been determined by $P_{j}$ and acts accordingly. If $p_{j}$ is scheduled to broadcast its own value on some channel $c$, then it transmits the vector of all values $m_{j, *}$ on channel $c$. If it is scheduled to broadcast as a surrogate for $p_{w}$ on some channel $c$, then $p_{j}$ transmits the vector of all the values $m_{w, *}$. If it is scheduled to receive on some channel $c$, then it does so. (In Section 5.6 we reduce the message size to constant.) When the round completes, at least one of the $t+1$ channels has transmitted successfully; the remaining $t$ may have been disrupted by the adversary. Notice that since $t+1$ honest nodes broadcast on the $t+1$ channels, then the adversary cannot successfully spoof messages, as every one of the $t+1$ channels on which nodes receive is in use.

\section{Feedback phase:}

In the second phase of simulating the move in the starrededge removal game, the nodes run communication-feedback, using the witness set $W$ as defined above. Each witness sets its flag as follows: if it received a message during the message transmission phase, then it sets its boolean flag to true; otherwise, it sets its flag to false. Notice that every witness for a given channel $c$ chooses the same setting for its flag. The communication-feedback routine returns to each process $p_{j}$ some set $D_{j}$. We will argue that, as per the guarantees of communication-feedback, every set $D_{j}$ is identical.

Each node $p_{j}$ then simulates the referee returning the following set of nodes and edges from $P_{j}$ : (1) every node $v \in P_{j}$ where $v$ was scheduled to broadcast during the messagetransmission round on channel $c$ and $c \in D_{j}$; (2) every edge $(w, z) \in P_{j}$ where $z$ was scheduled to receive on channel $c$ and $c \in D_{j} .{ }^{1}$

ThEOREM 6. f-AME is a t-disruptable Authenticated Message Exchange protocol that terminates in $O\left(|E| t^{2} \log n\right)$ rounds of communication.

Proof. We show that throughout the starred-edge removal game round simulation, the following three invariants are maintained:

\footnotetext{
${ }^{1}$ Notice that we could simplify the algorithm somewhat by having communication-feedback return the actual messages received on each non-disrupted channel, rather than simply the single bit of information indicating success or failure. The running time, however, would remain unchanged. We use the binary version of the communication-feedback as it seems from preliminary research to be more robust to harsher adversary models, including, for example, Byzantine node corruptions.
} 
(Invariant 1.) At the beginning of each simulated move of the starred-edge removal game, every node has the same simulated game graph $G$, and the same starred set $S$. This implies that the same nodes and edges have been removed in the game up until this round, and the same nodes have been added to $S$.

(Invariant 2.) For every node $p_{j} \in \Pi$, for every node $v \in$ $S_{j}$ (the starred-node set of node $p_{j}$ ), and for every $w$ such that $(v, w) \in E$ : the message $m_{v, w}$ is known to at least $3(t+1)$ nodes.

(Invariant 3.) After every simulated move of the starrededge removal game, for every process $p_{j}$, the graph $G_{j}$ represents the disruption graph for the AME problem. That is, if $(v, w) \in E^{A M E}$ and $w$ has not yet learned $m_{v, w}$, then edge $(v, w)$ is in $G_{j}$.

It is easy to see that all three invariants are initially true: Invariant 1 follows since $G_{j}$ and $S_{j}$ are initialized identically for every node $p_{j}$; Invariant 2 follows since $S_{j}$ is initially empty, for every node $p_{j}$; Invariant 3 follows since initially $G_{j}=G^{A M E}$ for every node $p_{j}$.

We proceed inductively to show that these invariants are maintained, with high probability, after each simulated move of the starred-edge removal game. We first note that since, at the beginning of the simulated round, every node $p_{j}$ has the same graph $G_{j}$ and the same set $S_{j}$, by Invariant 1, every node $p_{j}$ chooses the same set $P_{j}$ when executing the greedy-removal strategy. As a result, all the nodes calculate the same broadcast/receive schedule for the messagetransmission phase, and hence exactly one node broadcasts on each of the $t+1$ channels. This also implies that every node calculates the same witness set $W[c]$ for channel $c \in\{1, \ldots, C\}$.

We therefore conclude that each of the nodes scheduled to receive on some channel either receives a message from an honest transmitter, or receives nothing - in the case of an adversarial disruption. (Thus, adversarial spoofing is impossible here.) Since the adversary can disrupt at most $t$ channels, at least one set of nodes scheduled to listen does in fact receive a message in the message-transmission phase.

Moreover, we observe that every witness for a given channel $c \in\{1, \ldots, C\}$ receives the same message-or-silence during the message-transmission phase, and hence initiates the communicate-feedback routine with the same setting for its flag. Thus, we conclude by Lemma 5 that, with high probability, every node $p_{j}$ outputs $c \in D_{j}$ if and only if the broadcast on channel $c$ succeeded during the message transmission round. As a result, every node outputs the same set $D_{j}$, and $D_{j} \neq \emptyset$.

Since each node $p_{j}$ then simulates the referee in the same manner based on the same sets $D_{j}$ and $P_{j}$, we can conclude that each node $p_{j}$ updates its graph $G_{j}$ and its set $S_{j}$ in the same manner, maintaining Invariant 1.

Next, we argue that the second invariant is maintained: a node $v$ in the graph $G_{j}$ is added by node $p_{j}$ to set $S_{j}$ only if it was scheduled during the message transmission round to broadcast on some channel $c$, and only if $c \in D_{j}$ after the communication-feedback routine. This latter fact implies that the broadcast succeeded (as per Lemma 5), and hence that at least $3(t+1)$ witnesses received the message from $v$.

Finally, we note that the third invariant follows from the fact that a node $p_{j}$ removes edge $(v, w)$ from $G_{j}$ only if the channel $c$ on which $v$-or its surrogate-transmits $m_{v, w}$ to $w$ is not disrupted, i.e., if $c \in D_{j}$.

Having shown that each of the three invariants is maintained throughout the simulation, it remains to observe that this implies the correctness of the algorithm, and to calculate the running time.

First, note that for each of the $O(|E|)$ game rounds required by the greedy-removal strategy, the simulation succeeds with high probability. We conclude by a union bound over the $O(|E|)$ rounds that each round is simulated correctly with high probability.

Second, we notice that by Theorem 4 , at the end of $|E|$ simulated rounds, for every process $p_{j}$, the vertex cover for the graph $G_{j}$ is at most $t$. Invariant 3 implies an equivalence with the AME disruption graph, leading to the conclusion that f-AME satisfies $t$-disruptability. Finally, each simulated game round requires $O\left(t^{2} \log n\right)$ rounds of communication: communication-feedback requires $O(t \log n)$ rounds to deliver the feedback for each of $t+1$ channels, as described in Lemma 5. We conclude that the simulation of the $O(|E|)$ game rounds requires $O\left(|E| t^{2} \log n\right)$ rounds.

\subsection{Optimizing f-AME for More Channels}

For our analysis of $\mathrm{f}-\mathrm{AME}$, we assumed that $C=t+1$, the maximum value of $t$ for which any communication is possible. We have focused on this case in order to highlight that polynomial-time solutions exist even under worst-case interference. It is natural, however, to ask how performance might improve if more channels are available. Here we briefly consider two additional cases (the results are summarized in Figure 5.5).

\section{Case 1: $C \geq 2 t$.}

Assume that the protocol uses exactly $C=2 t$ of the available channels. In this case, each proposal in the starrededge removal game consists of $2 t$ elements, and the referee returns $t$ elements from each proposal. Thus, the greedy strategy terminates in $O(|E| / t)$ rounds. We next note that in each round of the communication-feedback routine, a listening node (that is not a witness) has a probability $\geq$ $1 / 2$ of avoiding disruption, and thus receives the feedback in $O(\log n)$ rounds with high probability. Thus, the total running time for acquiring feedback on all $2 t$ channels is $O(t \log n)$. As a result, the overall running time of the protocol is $O(|E| \log n)$.

Case 2: $C \geq 2 t^{2}$.

Fix $C^{\prime}=\lfloor C / t\rfloor$. In the message-transmission phase we use only $C^{\prime}$ of the available channels; we refer to these as the proposal channels. In the feedback phase, we use all $C$ channels. Thus, in the simulated starred-edge removal game, each proposal consists of $C^{\prime}$ elements; the referee responds with $C^{\prime}-t$ elements of the proposal. Thus, the greedy strategy now terminates in $O\left(|E| /\left(C^{\prime}-t\right)\right)=O(|E| / t)$ rounds.

The key, then, to obtaining better performance is to reduce the cost of feedback to $O\left(\log ^{2} n\right)$ rounds. Recall that for each channel, feedback can be accomplished in $O(\log n)$ rounds. Instead of executing the $O\left(C^{\prime}\right)$ instances of feedback sequentially (which would require $O(t \log n)$ time), we execute the feedback instances in parallel, using a parallelprefix tree to merge information until all the information is known to a single set of witness; these witness can then disseminate the feedback to everyone else. 


\begin{tabular}{|c|c|c|c|}
\hline Number of channels & greedy-removal & $\begin{array}{c}\text { communication-feedback } \\
\text { (per invocation) }\end{array}$ & f-AME \\
\hline \hline$C \geq t+1$ & $O(|E|)$ & $O\left(t^{2} \log n\right)$ & $O\left(|E| t^{2} \log n\right)$ \\
\hline$C \geq 2 t$ & $O\left(\frac{|E|}{t}\right)$ & $O(t \log n)$ & $O(|E| \log n)$ \\
\hline$C \geq 2 t^{2}$ & $O\left(\frac{|E|}{t}\right)$ & $O\left(\log ^{2} n\right)$ & $O\left(\frac{|E| \log ^{2} n}{t}\right)$ \\
\hline
\end{tabular}

Figure 3: Optimizing f-AME time complexity for larger number of channels.

In more detail, begin by pairing up the proposal channels, and assign each pair $\left(c_{1}, c_{2}\right)$ a unique set of $t$ channels. Using only the assigned channels, the witnesses for proposal channel $c_{1}$ use the communication-feedback routine to propagate information to the witnesses for proposal channel $c_{2}$. Next, conversely, witnesses for proposal channel $c_{2}$ use the communication-feedback routine to propagate information to the witnesses for proposal channel $c_{1}$. At this point, the witnesses are informed of the feedback for both channels $c_{1}$ and $c_{2}$. Each pair $\left\langle c_{1}, c_{2}\right\rangle$ is now assigned a companion pair $\left\langle c_{3}, c_{4}\right\rangle$, and the process continues merging information until there are $t+1$ witnesses that have all the feedback. A final instance of communication-feedback is used for these witnesses to propagate the feedback to everyone else. The total time for the modified feedback routine is now $O\left(\log n \log C^{\prime}\right)=O\left(\log ^{2} n\right)$. The running time for the entire game simulation is now $O\left(\frac{|E|}{t} \log ^{2} n\right)$.

\section{A Note on Optimality}

Notice that a trivial lower for authenticated message exchange is $\Omega\left(\frac{|E|}{C}\right)$. In particular, consider a graph $E$ consisting only of disjoint edges; at most $C$ of the sources can transmit information in each round. When $C \geq 2 t$, the optimized $\mathrm{f}-\mathrm{AME}$ algorithm is within a factor of $t \log n$ of achieving this lower bound; when $C \geq 2 t^{2}$, it is within a factor of $t \log ^{2} n$. Closing this gap, perhaps by deriving a non-trivial lower bound, remains an open question.

\subsection{Optimizing f-AME Message Size}

Each message of the f-AME protocol may be quite large. In fact, each message sent by process $p_{v}$ may include up to $n-1$ messages of the form $m_{v, *}$. Here we describe an optimization that reduces the size of a protocol message to include no more than a constant number of $\mathrm{AME}$ values.

The optimized protocol is divided into two parts. In the first part, the message gossip phase, nodes exchange messages using a randomized gossip protocol. This ensures that every node has received every message; however it does not guarantee any authentication. In the second part, the original $\mathrm{f}$-AME protocol is used to exchange shorter digests that can be use to authenticate the true messages from the gossip phase. In order to accomplish this, we will rely on secure hash functions. Let $H_{1}$ and $H_{2}$ be two such functions. Fix an edge set $E$, and let $E_{v} \subseteq E$ be the edges of the form $(v, *)$. Let $M_{v}$ be an ordered sequence of the values to be sent on the edges in $E_{v}$.

\section{Message Gossip Phase}

Each edge $(v, w) \in E$ is given one epoch of length $\Theta\left(t^{2} \log n\right)$ during which $v$ communicates $m_{v, w}$. To reduce the number of fake messages assumed to be potentially true, nodes append some extra digest information onto each of these transmissions. Specifically, fix some node $v \in \Pi$, and let $\left\{m_{v, 1}, \ldots, m_{v, k}\right\}=M_{v}$. Assume $M_{v}$ describes the order of the epochs for these messages, that is, the epoch for $(v, 1)$ precedes the epoch for $(v, 2)$, and so on. For the $(v, i)$ epoch, process $v$ broadcasts the following information on a randomly chosen channel for each of the $\Theta\left(t^{2} \log n\right)$ rounds: the message $m_{v, i}$, along with the reconstruction hash defined as $H_{1}\left(m_{v, i}, \ldots, m_{v, k}\right)$. All other nodes simply choose a random channel on which to listen, and remember what they have received. By the end of each epoch $(v, i)$, with high probability, every node has received $v$ 's transmission (as per a straightforward Chernoff bound and a union bound over the $n-1$ nodes). They may also have (potentially) received many spoofed messages.

\section{Reconstruction Phase}

The message gossip phase concludes with each node attempting to reconstruct $M_{v}$ for each $v \in \Pi$. Having received $O\left(t^{2} \log n\right)$ messages during each epoch for $v$, there may be exponentially many possible $M_{v}$ vectors. The reconstruction hash allows us to reduces these to a polynomial number of possibilities. To perform the reconstruction for $v \in \Pi$, we arrange the received messages into levels $1, \ldots, k$. In each level $i$ we place all messages received during $v$ 's $i^{t h}$ epoch. We will attempt to decorate these levels with directed edges. Each directed edge will connect messages at some level $i$ to messages at level $i+1$.

The decoration proceeds backwards as follows, beginning with level $k$, the largest level: For each message $m_{1}$ at level $k-1$, and for each message $m_{2}$ at level $k$, calculate the hash of each sequence consisting of message $m_{1}$, followed by message $m_{2}$. Draw an edge from message $m_{1}$ to message $m_{2}$ if and only if the reconstruction hash tagged on message $m_{1}$ equals $H_{1}\left(m_{1}, m_{2}\right)$.

We repeat this procedure as we move down the levels. For each level $i$ message $m_{1}$, consider the hash produced by each of the sequences consisting of a level $i+1$ message, followed by a chain of messages reached by following outgoing edges up to level $k$; create an edge between $m_{1}$ and the level $i+1$ message when the calculated hash matches the reconstruction hash attached to the level $i$ message. 
The procedure requires $O\left(t^{4} \log ^{2} n\right)$ hashes for each of the $k-1$ levels. Assuming a collision-resilient hash function, each message will be decorated with a most one outgoing edge. (Recall, however, that local computation is relatively cheap compared to the cost of sending messages.) It follows that the resulting decorated levels will include at most $O\left(t^{2} \log n\right)$ chains of messages, each described by a path from level 1 to level $k$. Among these, of course, will be one sequence that actually represents $M_{v}$.

\section{The Vector Signature}

In order to identify the correct sequence of messages and thus ensure authenticity, we use the f-AME protocol with the following modification: each message containing $M_{v}$ is replaced with the constant-sized message $H_{2}\left(M_{v}\right)$, the vector signature for $v$. Whenever a node first receives the vector signature for $v$, it can compare it to the $\mathrm{H}_{2}$ hashes of its $O\left(t^{2} \log n\right)$ validly reconstructed $M_{v}$ vectors from the reconstruction phase, and thus validate the single vector that is correct. Of course, once it knows the real $M_{v}$, it can extract any message from $v$ required by $\mathrm{f}-\mathrm{AME}$. The authentication guarantee of $f-A M E$ ensures that the received vector signature is authentic.

\section{A SHARED GROUP KEY}

We now describe how to use f-AME to establish a secret key shared among all but at most $t$ nodes that is unknown to the adversary. Group keys have a variety of uses from broadcast encryption to group management. In section Section 7 , we describe one possible use in the setup of a long-lived, reliable, secure, and authenticated communication service.

\section{Part 1: Initialize Shared Keys}

We begin by choosing a set of $t+1$ leader nodes, and we establish a shared secret key between each leader node and each non-leader node. Let $\ell \subset \Pi$ contain $t+1$ leader nodes, and define the pair set $E_{\ell}=\{(v, w) \mid v \in \ell \vee w \in \ell\}$.

We then use f-AME to swap messages for a one-round key exchange protocol, e.g., Diffie-Hellman Key Exchange (DHKE) [12]. Specifically, execute f-AME where $E=E_{\ell}$ and each message $m_{v, w}$ is the appropriate DHKE message to be sent from $v$ to $w$. Any non-disrupted process pair can use the DHKE messages to establish a shared key unknown to the adversary. ${ }^{2}$ The total cost: $O\left(n t^{3} \log n\right)$ rounds.

\section{Part 2: Disseminate Leader Keys}

Recall that f-AME guarantees sender awareness, and thus each leader knows how many shared secrets were successfully established. If a leader exchanges messages with at least $n-t$ nodes, it considers itself complete. Each complete leader chooses a leader key $K_{v}$.

We assign an epoch of length $\Theta(t \log n)$ for each pair $(v, w)$ where $v \in \ell, w \in \Pi-\{v\}$. If $v$ and $w$ failed to establish a shared secret, they remain silent during their epoch. Otherwise, using their shared secret, they calculate a pseudorandom channel-hopping pattern that is unknown to the adversary. During each round of the epoch, if $v$ is complete, it transmits the leader key $K_{v}$, encrypted with the key

\footnotetext{
${ }^{2}$ The specific security assumption for DHKE holds that calculating the shared key can be reduced to the Computational Diffie-Hellman assumption; that is, calculating discrete log in a large finite field.
}

shared by $v$ and $w$ on the calculated pseudo-random channel. Otherwise, it sends the message "incomplete." Node $w$ listens accordingly. The pair avoid interference in each round with probability at least $1 /(t+1)$. Therefore, over $\Theta(t \log n)$ rounds, we conclude that $w$ receives the leader key with high probability. The total cost: $\Theta\left(n t^{2} \log n\right)$ rounds.

\section{Part 3: Key Agreement}

Finally, the nodes collectively choose one of the leader keys to be the shared group key. Let $S$ be a collection of $2 t+1$ non-leader nodes. We assign an epoch of length $\Theta\left(t^{2} \log n\right)$ to each node $i \in S$. During this epoch, node $i$ randomly chooses channels on which to broadcast the identity of the smallest leader $j$ from which it received a key $K_{j}$ during Part 2, and a hash of the key $K_{j}$. Other nodes receive on random channels. Because epochs lasts $\Theta\left(t^{2} \log n\right)$ rounds, every node should hear from all nodes in $S$.

After the epochs conclude, we apply a simple agreement rule: If a node heard at least $t+1$ non-leader nodes report the same leader, and the node can verify each of these messages (i.e., it knows the leader key for this leader and can therefore confirm the leader key hash for these reports), then it adopts this leader key as the group key. Otherwise, it recognizes that it does not know the group key.

To see that this works, let $j$ be the smallest completed leader. We know that at least $t+1$ nodes in $S$ heard from $j$ in Part 2, since $j$ is complete, and thus report $j$ in Part 3. (Recall that incomplete leaders do not broadcast in Part 2.) Therefore, the $n-t$ nodes that received $j$ 's leader key $K_{j}$ confirm the reports and adopt $K_{j}$. (The other $t$ nodes will correctly identify their lack of knowledge.) The total cost of this part: $\Theta\left(t^{3} \log n\right)$ rounds. The total cost of the entire protocol is dominated by Part 1: $\Theta\left(n t^{3} \log n\right)$ rounds. (With more channels, the cost can be reduced accordingly.)

\section{LONG-LIVED COMMUNICATION}

Once we have established shared secret keys, we can construct a long-lived communication protocol that simulates a secure channel. Thus nodes can execute the f-AME protocol once to bootstrap the secure channel emulation, and from then on out, rely on this more efficient and robust communication primitive. The long-lived communication protocol guarantees, with high probability: (1) $t$-Reliability: all but $t$ nodes can communicate on the emulated channel; (2) Secrecy: the adversary cannot eavesdrop on the channel; and (3) Authentication: a node $w$ receives a message $m$ from $v$ only if $v$ previously sent message $m$.

Such a primitive is easy to implement using a shared secret group key $K$, as established in Section 6 . The nodes generate a channel-hopping pattern by using $K$ as the seed to a pseudo-random generator. The nodes then participate in each round on the specified channel.

To broadcast in an emulated round, a node repeats its message, encrypted using key $K$, for $\Theta(t \log n)$ rounds; other nodes simply listen. Since the channel-hopping appears random to the adversary, it is easy to see that if only one node broadcasts, its message will be received, without disruption, with high probability. (As before, for $C \geq 2 t$, the number of required real rounds would fall to $O(\log n)$.) If two or more nodes broadcast concurrently, then, as on a real broadcast channel, one or more messages might be lost. The channel emulation requires $\Theta\left(n t^{3} \log n\right)$ rounds to setup the group key, and each emulated round requires $\Theta(t \log n)$ rounds. 


\section{OPEN QUESTIONS}

By focusing on multi-channel wireless communication, this paper introduces a new environment for the study of secure communication. Thus, there are a variety of open questions to consider. (1) Byzantine corruption: can we tolerate corruption faults, where the set of honest, participating devices is unknown? A simple modification allows us to achieve $2 t$ disruptability in this case: surrogates are eliminated, and every rumor is received directly from its source; $t+1$ nodes are used to report on every non-disrupted channel during communication-feedback. However, it remains open whether $t$-disruptability can be achieved. (2) Information-theoretic security: the secrecy of the shared keys here depends on cryptographic assumptions, such as (computational DiffieHellman). We can restrict the adversary to listening on only $t$ channels, so at least one channel's communication is unknown. In this case, is it possible to achieve some shared secrets that are secure in an information-theoretic sense? We conjecture that any such algorithm in inherently exponential in time complexity. (3) Beyond $t$-disruptability: is it possible to make some progress with the disrupted nodes, even if it is at the cost of weakening, for them, some of the AME guarantees? (4) Point-to-point communication and beyond: do there exist more efficient point-to-point primitives? What other communication and coordination activities are practical? (5) Multi-hop networks: all of these questions can be studied in the context of multi-hop networks.

\section{REFERENCES}

[1] N. Alon, A. Bar-Noy, N. Linial, and D. Peleg. A lower bound for radio broadcast. Journal of Computer and System Sciences, 43(2):290-298, October 1992.

[2] R. Bar-Yehuda, O. Goldreich, and A. Itai. On the time-complexity of broadcast in multi-hop radio networks: An exponential gap between determinism and randomization. Journal of Computer and System Sciences, 45(1):104-126, 1992.

[3] R. Bar-Yehuda, A. Israeli, and A. Itai. Multiple communication in multi-hop radio networks. SIAM Journal on Computing, 22(4):875-887, 1993.

[4] V. Bhandari and N. H. Vaidya. On reliable broadcast in a radio network. In the Proceedings of the International Symposium on Principles of Distributed Computing, pages 138-147, July 2005.

[5] Bluetooth Consortium. Bluetooth Specification Version 2.1, July 2007.

[6] M. Cagalj, S. Capkun, and J-P. Hubaux. Key agreement in peer-to-peer wireless networks. Proceedings of the IEEE (Special Issue on Cryptography and Security), 94(2), February 2006.

[7] B. S. Chlebus, L. Gasieniec, A. Lingas, and A. T. Pagourtzis. Oblivious gossiping in ad-hoc radio networks. In the Proceedings of the 5th International Workshop on Discrete Algorithms and Methods for Mobile Computing and Communications, pages 44-51, 2001.

[8] B.S. Chlebus and D.R. Kowalski. Robust gossiping with an application to consensus. Journal of Computer and System Sciences, 72(8):1262-1281, December 2006.

[9] A. Clementi, A. Monti, and R. Silvestri. Optimal f-reliable protocols for the do-all problem on single-hop wireless networks. Algorithms and Computation, pages 320-331, 2002.

[10] A. Clementi, A. Monti, and R. Silvestri. Round robin is optimal for fault-tolerant broadcasting on wireless networks. Journal of Parallel and Distributed Computing, 64(1):89-96, 2004.

[11] Artur Czumaj and Wojciech Rytter. Broadcasting algorithms in radio networks with unknown topology. In the Proceedings of the Symposium on Foundations of Computer Science, October 2003.

[12] W. Diffe and M. Hellman. New directions in cyptography. Transactions on Information Theory, November 1976.

[13] S. Dolev, S. Gilbert, R. Guerraoui, and C. Newport. Gossiping in a multi-channel radio network: An oblivious approach to coping with malicious interference. In the Proceedings of the International Symposium on Distributed Computing, September 2007.

[14] S. Gilbert, R. Guerraoui, and C. Newport. Of malicious motes and suspicious sensors: On the efficiency of malicious interference in wireless networks. In the Proceedings of the International Conference on Principles of Distributed Systems, December 2006.

[15] J. Komlos and A.G. Greenberg. An asymptotically fast non-adaptive algorithm for conflict resolution in multiple access channels. IEEE Transactions of Information Theory, 31(2,) March 1985.

[16] C-Y. Koo. Broadcast in radio networks tolerating byzantine adversarial behavior. In the Proceedings of the International Symposium on Principles of Distributed Computing, pages 275-282, July 2004.

[17] C-Y. Koo, V. Bhandari, J. Katz, and N. H. Vaidya. Reliable broadcast in radio networks: The bounded collision case. In the Proceedings of the International Symposium on Principles of Distributed Computing, July 2006.

[18] D. Kowalski and A. Pelc. Time of deterministic broadcasting in radio networks with local knowledge. SIAM Journal on Computing, 33(4):870-891, 2004.

[19] E. Kranakis, D. Krizanc, and A. Pelc. Fault-tolerant broadcasting in radio networks. Journal of Algorithms, 39(1):47-67, April 2001.

[20] E. Kushelevitz and Y. Mansour. An $\Omega(d \log (n / d))$ lower bound for broadcast in radio networks. In the Proceedings of the International Symposium on Principles of Distributed Computing, August 1993.

[21] A. Pelc and D. Peleg. Feasibility and complexity of broadcasting with random transmission failures. In the Proceedings of the International Symposium on Principles of Distributed Computing, pages 334-341, July 2005.

[22] F. Stajano and R. Anderson. The resurrecting duckling: security issues for ad hoc wireless networks. In the Proceedings of the International Workshop on Security Protocols, April 1999.

[23] D. E. Willard. Log-logarithmic selection resolution protocols in a multiple access channel. SIAM Journal of Computing, 15(2):468-477, 1986. 